\title{
Review Article \\ Effect of Zirconia Dental Implant Surfaces on Bone Integration: A Systematic Review and Meta-Analysis
}

\author{
Ali Hafezeqoran and Roodabeh Koodaryan \\ Department of Prosthodontics, Faculty of Dentistry, University of Medical Sciences, Tabriz, Iran \\ Correspondence should be addressed to Roodabeh Koodaryan; koodaryan@gmail.com
}

Received 18 September 2016; Accepted 11 January 2017; Published 16 February 2017

Academic Editor: Despina Deligianni

Copyright (c) 2017 Ali Hafezeqoran and Roodabeh Koodaryan. This is an open access article distributed under the Creative Commons Attribution License, which permits unrestricted use, distribution, and reproduction in any medium, provided the original work is properly cited.

\begin{abstract}
Background. The information available about osseointegration and the bone to implant interaction of zirconia implants with various surface modifications is still far from sufficient. Objective. The purpose of this systematic review and meta-analysis was to evaluate and compare zirconia dental implants with different surface topographies, with a focus on bone to implant contact and removal torque. Methods. The systematic review of the extracted publications was performed to compare the bone to implant contact (BIC) with removal torque (RT) values of titanium dental implants and machined and surfaced modified zirconia implants. Results. A total of fifteen articles on BIC and RT values were included in the quantitative analysis. No significant difference in the BIC values was observed between titanium and machined zirconia implants ( $p=0.373$; 95\% CI: -0.166 to 0.443 ). However, a significantly better BIC values were observed for acid etched zirconia implants compared with those of titanium implants $(p=0.032$; $95 \%$ CI: 0.068 to 1.461$)$. Unmodified zirconia implants showed favorable BIC values compared to modified-surface zirconia implants $(p=0.021 ; 95 \%$ CI: -0.973 to -0.080$)$. Conclusion. Acid etched zirconia implants may serve as a possible substitute for successful osseointegration.
\end{abstract}

\section{Introduction}

Commercially pure titanium and some of its alloys have so far been the material of choice in implant dentistry and orthopedics [1]. However, the gray color of titanium impairs esthetic results particularly in the presence of thin mucosal biotype [2]. In recent years, high strength zirconia implants have emerged as an alternative to titanium implants and provide better esthetic outcomes [3]. Yttrium-stabilized tetragonal zirconia is a well-studied bioinert structure which provides high strength, fracture toughness, esthetic, and biocompatibility [4].

Osseointegration is biological fixation of implant relating to direct bone to implant contact (BIC) without an intervening connective tissue layer [5]. BIC is regarded as key indicator for successful osseointegration which governs the overall success and survival of implants [6]. Moreover, it is clearly understood that the surface properties of a biomaterial play a fundamental role in osseointegration process
[7]. Implant material composition and surface topography influence the wound healing processes following the implantation and subsequently affect osseointegration [8]. A moderately rough surface topography is known to positively affect the interfacial tissue reaction [9]. Therefore, numerous surface modification methods have been proposed to enhance osseointegration and improve the success rates. Such approaches mainly include optimization of the surface microroughness (sandblasting or acid etching), application of the bioactive coatings (calcium phosphate, bisphosphonate, and collagen), sintering particles onto the implant surface, nanotechnology, and laser technology [10-14]. Although there has been considerable discussion of zirconia surface modification and structure, the information available about osseointegration responses and the implant-bone interaction of these implants is still far from sufficient. Thus, the optimal surface topography for a dental implant remains unclear [15].

The present systematic review and meta-analysis was conducted to evaluate BIC around zirconia implants with 
different surfaces. The null hypotheses are as follows. (1) There are no differences in BIC for machined zirconia dental implants compared with titanium implants and (2) there are no differences among zirconia dental implants with different surface modifications with regard to BIC and RT.

\section{Methods}

2.1. Structure of the Review. This systematic review adheres to the criteria of the "Preferred Reporting Items for Systematic Review and Meta-Analysis" (PRISMA) [16].

2.2. Search Strategy. Electronic searches without time restrictions were performed in the PubMed database for relevant publications until 15 June 2016. The following search terms were used in this study: dental implant, zirconia implant, zirconia dental implant, zirconia osseointegration, removal torque values, histomorphometry, zirconia surface treatment, and dental implant surface treatment with OR and AND. Authors also manually searched the literature for relevant publications in British Journal of Oral and Maxillofacial Surgery, Clinical Implant Dentistry and Related Research, Clinical Oral Implants Research, European Journal of Oral Implantology, Implant Dentistry, International Journal of Oral and Maxillofacial Implants, International Journal of Oral and Maxillofacial Surgery, International Journal of Periodontics and Restorative Dentistry, International Journal of Prosthodontics, Journal of Clinical Periodontology, Journal of Dental Research, Journal of Dentistry, Journal of Oral Implantology, Journal of Craniofacial Surgery, Journal of Cranio-Maxillofacial Surgery, Journal of Maxillofacial and Oral Surgery, Journal of Oral and Maxillofacial Surgery, and Journal of Periodontology.

2.3. Eligibility Criteria. The studies were included if they met the following inclusion criteria: (1) animal studies, (2) publication in the international peer reviewed literature, (3) English language, and (4) histological (animals) assessment of BIC and RT.

Exclusion criteria were (1) animal studies with less than two animals per observation or group, (2) computational studies, (3) human studies, (4) studies that evaluated only one type of implant surface, and (5) reviews.

Based on population, intervention, control, and outcome (PICO) criteria, the focused questions were as follows. (1) Does the choice of implant material affect bone to implant contact when comparing titanium to zirconia in animal models? (2) What is the impact of zirconia implant surface modification on BIC and RT when comparing modified to as sintered zirconia in animal models? The study population was the animals which received machined or surface-modified zirconia and titanium dental implants. The comparison was made between titanium and machined zirconia, between titanium and surface-modified zirconia implants, and between machined and surface-modified zirconia implants. The two outcomes evaluated were the BIC and RT of implants.

2.4. Study Selection. The titles were screened independently by the two reviewers. The abstracts of all studies were assessed for relevance to the review and those appearing to meet the inclusion criteria were retrieved. Also, all reference lists of the selected studies and relevant reviews were checked manually to identify additional articles that have been missed in database searches. Disagreements were settled by discussion between the authors until a consensus was achieved.

2.5. Quality Assessment. All studies were assessed for quality depending on whether they met all the quality criteria or if one or more criteria were partially met or not met using the SYRCLE's risk of bias tool [17]. This tool contains 10 entries which facilitates bias judgment. "Yes" indicates a low risk of bias and "no" indicates a high risk of bias, while" unclear" means insufficient information to assess the risk of bias.

2.6. Summary Measures and Synthesis of Results. In the majority of included studies, the animals were subdivided into various groups. The comparison was performed for different implant materials and surface treatments. These data were recorded separately during the review to be incorporated into the analysis. Thus the population of the study was more than indicated by the numbers of the included studies.

The meta-analysis was based on the Mantel-Haenzel and Inverse Variance methods. Bone to implant contact and the removal torque were the continuous outcome measures which were expressed in standard difference in mean. A fixed model was used to calculate the weighted means at 95\% confidence intervals (CI). The values were considered significant when $p<0.05$. The data were analyzed using comprehensive meta-analysis software version 2 (Biostat Inc., Englewood, New Jersey, USA).

\section{Results}

3.1. Literature Search. The search in the database retrieved an initial number of 2018 references. 1900 titles were excluded after further assessment (Figure 1). All selected publications were subdivided according to differences in implant materials and surface treatments into 3 groups: (1) studies that assessed the impact of the machined or surface-modified zirconia implant on BIC in comparison to titanium implants, (2) studies that evaluated the BIC of powder injection mold (PIM) zirconia implants, and (3) studies that compared RT of machined zirconia with surface-modified zirconia implants (Table 1). After the application of the inclusion/exclusion criteria and qualitative assessment of these publications, 15 studies were considered for a quantitative meta-analysis (Tables 1 and 2).

3.2. Meta-Analysis. A total of 15 studies were included in this quantitative meta-analysis which were published from 2004 to 2015 . Seven of the selected studies $[14,18-23]$ evaluated the $\mathrm{BIC}$ values of machined zirconia and titanium surfaces. Three studies compared BIC values of titanium and etched zirconia implants $[6,24,25]$ and 3 other studies [14, 20, 26] assessed those of titanium and blasted zirconia implants. Machined and surface-modified zirconia implants were considered in 3 studies [14, 18, 21]. PIM-treated and untreated zirconia 
TABLE 1: Characteristics of studies included in review.

\begin{tabular}{|c|c|c|c|c|c|c|c|}
\hline Study, year & Animal & $\begin{array}{l}\text { Implant no, } \\
\text { dimensions } \\
\left(\mathrm{mm}^{2} \times \mathrm{mm}\right)\end{array}$ & Manufacturer & Material & $\begin{array}{l}\text { Surface } \\
\text { treatment }\end{array}$ & BIC (\%) & $\begin{array}{c}\mathrm{RT} \\
(\mathrm{N} / \mathrm{cm})\end{array}$ \\
\hline \multirow{5}{*}{$\begin{array}{l}\text { (Thoma et al. } \\
\text { 2015) }\end{array}$} & \multirow{5}{*}{ Dog } & 48 & & & & 6 months: & \\
\hline & & $4 \times 8$ & $\begin{array}{c}\text { VITA } \\
\text { Zahnfabrik }\end{array}$ & $\begin{array}{c}\text { One piece } \\
\mathrm{Zr}\end{array}$ & - & $\begin{array}{c}87.71 \pm \\
25.07\end{array}$ & - \\
\hline & & $3.7 \times 9$ & Metoxit & $\begin{array}{c}\text { One piece } \\
\mathrm{Zr}\end{array}$ & Microporous & $\begin{array}{c}78.58 \pm \\
17.26\end{array}$ & - \\
\hline & & $4.1 \times 8$ & BPI & $\begin{array}{c}\text { Two piece } \\
\mathrm{Zr}\end{array}$ & $\begin{array}{l}\text { Nanostructured } \\
\text { and hydrophilic }\end{array}$ & $\begin{array}{c}84.17 \pm \\
25.07\end{array}$ & - \\
\hline & & $3.3 \times 8$ & Straumann & $\begin{array}{c}\text { One piece } \\
\mathrm{Ti}\end{array}$ & $\begin{array}{c}\text { Sandblasted and } \\
\text { etched }\end{array}$ & $\begin{array}{c}87.85 \pm \\
13.59 \\
\end{array}$ & - \\
\hline \multirow{3}{*}{$\begin{array}{l}\text { (Kim et al. } \\
\text { 2015) }\end{array}$} & \multirow{3}{*}{ Rabbit } & & & & & 4 weeks: & \\
\hline & & 64 & Dentime & $\mathrm{Zr}$ & Machined & $\begin{array}{c}32.15 \pm \\
10.76\end{array}$ & 19.44 \\
\hline & & $4 \times 7$ & Cetatech & $\mathrm{Zr}$ & PIM roughened & $\begin{array}{c}58.38 \pm \\
11.28 \\
\end{array}$ & 57.63 \\
\hline \multirow{4}{*}{$\begin{array}{l}\text { (Park et al. } \\
\text { 2013) }\end{array}$} & \multirow{4}{*}{ Rabbit } & & & & & 4 weeks: & \\
\hline & & 80 & Chaorum & $\mathrm{Ti}$ & Machined & $\begin{array}{l}42.54 \pm \\
10.26\end{array}$ & $\begin{array}{l}10.56 \pm \\
6.03\end{array}$ \\
\hline & & $4 \times 7$ & Cetatech & $\mathrm{Zr}$ & PIM untreated & $\begin{array}{c}61.63 \pm \\
12.39\end{array}$ & $\begin{array}{c}44.24 \pm \\
8.41\end{array}$ \\
\hline & & & Cetatech & $\mathrm{Zr}$ & PIM roughened & $\begin{array}{c}64.42 \pm \\
11.45 \\
\end{array}$ & $\begin{array}{l}64 \pm 35 \\
\pm 10.46 \\
\end{array}$ \\
\hline \multirow{3}{*}{$\begin{array}{l}\text { (Chung et al. } \\
\text { 2013) }\end{array}$} & \multirow{3}{*}{ Rabbit } & 100 & & & & 4 weeks: & \\
\hline & & $4 \times 7$ & Cetatech & $\mathrm{Zr}$ & PIM untreated & $\begin{array}{c}59.59 \pm \\
11.50\end{array}$ & $\begin{array}{l}45 \pm 63 \\
\pm 10.78\end{array}$ \\
\hline & & & Cetatech & $\mathrm{Zr}$ & PIM roughened & $\begin{array}{c}61.52 \pm \\
12.96 \\
\end{array}$ & $\begin{array}{c}64.99 \pm \\
12.21\end{array}$ \\
\hline \multirow{4}{*}{$\begin{array}{l}\text { (Shon et al. } \\
\text { 2014) }\end{array}$} & \multirow{4}{*}{ Rabbit } & 100 & Cetatech & $\mathrm{Zr}$ & PIM untreated & $\begin{array}{c}4 \text { weeks: } \\
58.26 \pm \\
10.09\end{array}$ & $\begin{array}{c}39.7 \pm \\
11.69\end{array}$ \\
\hline & & $4 \times 7$ & Cetatech & $\mathrm{Zr}$ & PIM roughened & $\begin{array}{c}56.93 \pm \\
12.95\end{array}$ & $\begin{array}{c}59.21 \pm \\
12.35\end{array}$ \\
\hline & & & Cetatech & $\mathrm{Zr}$ & $\begin{array}{c}\text { PIM untreated + } \\
\text { He plasma }\end{array}$ & $\begin{array}{c}70.87 \pm \\
9.11\end{array}$ & $\begin{array}{c}46.75 \pm \\
13.15\end{array}$ \\
\hline & & & Cetatech & $\mathrm{Zr}$ & $\begin{array}{c}\text { PIM roughened } \\
+ \text { He plasma }\end{array}$ & $\begin{array}{c}72.27 \pm \\
10.31\end{array}$ & $\begin{array}{c}60.98 \pm \\
12.70 \\
\end{array}$ \\
\hline \multirow{6}{*}{$\begin{array}{l}\text { (Gahlert et al. } \\
\text { 2012) }\end{array}$} & \multirow{6}{*}{ Minipig } & 36 & Straumann AG & $\mathrm{Zr}$ & $\begin{array}{l}\text { Hydrofluoric } \\
\text { acid }\end{array}$ & $\begin{array}{l}4 \text { weeks: } \\
70 \pm 14.5\end{array}$ & - \\
\hline & & $4.1 \times 10$ & & & & $\begin{array}{l}8 \text { weeks: } \\
67.1 \pm 21.1\end{array}$ & - \\
\hline & & & & & & $\begin{array}{l}12 \text { weeks: } \\
68.3 \pm 22.8\end{array}$ & - \\
\hline & & & Straumann AG & $\mathrm{Ti}$ & SLA & $\begin{array}{l}4 \text { weeks: } \\
64.7 \pm 9.4\end{array}$ & - \\
\hline & & & & & & $\begin{array}{l}8 \text { weeks: } \\
68.3 \pm 22.8\end{array}$ & - \\
\hline & & & & & & $\begin{array}{c}12 \text { weeks } \\
83.7 \pm 10.3 \\
\end{array}$ & - \\
\hline \multirow{3}{*}{$\begin{array}{l}\text { (Montero et } \\
\text { al. 2015) }\end{array}$} & \multirow{3}{*}{ Dog } & 32 & & & & 5 months & \\
\hline & & $3.8 \times 8$ & Metoxit AG & $\mathrm{Zr}$ & Machined & $57 \pm 15.2$ & - \\
\hline & & & Microdent & $\mathrm{Ti}$ & - & $56.5 \pm 14.4$ & - \\
\hline
\end{tabular}


TABle 1: Continued.

\begin{tabular}{|c|c|c|c|c|c|c|c|}
\hline Study, year & Animal & $\begin{array}{l}\text { Implant no, } \\
\text { dimensions } \\
\left(\mathrm{mm}^{2} \times \mathrm{mm}\right)\end{array}$ & Manufacturer & Material & $\begin{array}{l}\text { Surface } \\
\text { treatment }\end{array}$ & BIC (\%) & $\begin{array}{c}\mathrm{RT} \\
(\mathrm{N} / \mathrm{cm})\end{array}$ \\
\hline \multirow{8}{*}{$\begin{array}{l}\text { (Hoffmann et } \\
\text { al. 2012) }\end{array}$} & \multirow{8}{*}{ Rabbit } & 96 & $Z$-system AG & $\mathrm{Zr}$ & Machined & $\begin{array}{c}6 \text { weeks: } \\
32.996 \pm \\
14.192\end{array}$ & $\begin{array}{r}35.409 \\
\pm 9.063\end{array}$ \\
\hline & & $3.25 \times 6$ & & & & $\begin{array}{c}12 \text { weeks: } \\
33.746 \pm \\
14.529\end{array}$ & $\begin{array}{c}40.591 \pm \\
17.081\end{array}$ \\
\hline & & & $Z$-system AG & $\mathrm{Zr}$ & Laser modified & $\begin{array}{c}6 \text { weeks: } \\
39.965 \pm \\
13.194\end{array}$ & $\begin{array}{r}26.309 \\
\pm 11.415\end{array}$ \\
\hline & & & & & & $\begin{array}{c}12 \text { weeks: } \\
43.87 \pm \\
14.544\end{array}$ & $\begin{array}{l}39.708 \\
\pm 9.819\end{array}$ \\
\hline & & & $Z$-system AG & $\mathrm{Zr}$ & Sandblasted & $\begin{array}{c}6 \text { weeks: } \\
39.614 \pm \\
15.029\end{array}$ & $\begin{array}{c}19.590 \pm \\
12.128\end{array}$ \\
\hline & & & & & & $\begin{array}{c}12 \text { weeks: } \\
41.350 \pm \\
15.816\end{array}$ & $\begin{array}{c}28.727 \\
\pm 18.766\end{array}$ \\
\hline & & & - & $\mathrm{Ti}$ & Acid etched & $\begin{array}{c}6 \text { weeks: } \\
34.155 \pm \\
15.816\end{array}$ & $\begin{array}{c}39.818 \pm \\
14.093\end{array}$ \\
\hline & & & & & & $\begin{array}{c}12 \text { weeks: } \\
34.818 \pm \\
12.209\end{array}$ & $\begin{array}{c}51.909 \pm \\
16.149\end{array}$ \\
\hline \multirow{6}{*}{$\begin{array}{l}\text { (Aboushelib } \\
\text { et al. 2013) }\end{array}$} & \multirow{6}{*}{ Rabbit } & 60 & - & $\mathrm{Zr}$ & Machined & $\begin{array}{l}4 \text { weeks: } \\
53.30 \pm 4.2\end{array}$ & - \\
\hline & & $3.7 \times 8$ & & & & $\begin{array}{l}6 \text { weeks: } \\
62.14 \pm 2.8\end{array}$ & - \\
\hline & & & Zimmer Dental & $\mathrm{Ti}$ & SLA & $\begin{array}{l}4 \text { weeks: } \\
56.93 \pm 3.9\end{array}$ & - \\
\hline & & & & & & $\begin{array}{l}6 \text { weeks: } \\
68.31 \pm 4.2\end{array}$ & - \\
\hline & & & - & $\mathrm{Zr}$ & $\begin{array}{c}\text { Selective } \\
\text { infiltration }\end{array}$ & $\begin{array}{l}4 \text { weeks: } \\
65.38 \pm 5.7\end{array}$ & - \\
\hline & & & & & etching & $\begin{array}{c}6 \text { weeks: } \\
75.01 \pm 5.1\end{array}$ & - \\
\hline \multirow{5}{*}{$\begin{array}{l}\text { (Koch et al. } \\
2010)\end{array}$} & \multirow{5}{*}{ Dog } & 48 & & & & 4 months: & \\
\hline & & & - & $\mathrm{Zr}$ & Machined & $59.11 \pm 7.45$ & - \\
\hline & & & - & $\mathrm{Zr}$ & $\mathrm{TiO} 2$ coated & $\begin{array}{c}55.83 \pm \\
13.92\end{array}$ & - \\
\hline & & & - & $\mathrm{Ti}$ & Sandblasted & $\begin{array}{c}40.91 \pm \\
10.11\end{array}$ & - \\
\hline & & & - & Peek & - & $26 \pm 8.9$ & - \\
\hline \multirow{3}{*}{$\begin{array}{l}\text { (Shin et al. } \\
\text { 2011) }\end{array}$} & \multirow{3}{*}{ Rabbit } & 20 & & & & 6 weeks: & \\
\hline & & $3.5 \times 6.6$ & - & $\mathrm{Zr}$ & Machined & $26 \pm 17.2$ & $\begin{array}{c}18.2 \pm \\
2.69\end{array}$ \\
\hline & & & - & $\mathrm{Ti}$ & Machined & $35.8 \pm 21.8$ & $\begin{array}{c}10.9 \pm \\
7.82\end{array}$ \\
\hline
\end{tabular}


TABle 1: Continued.

\begin{tabular}{|c|c|c|c|c|c|c|c|}
\hline Study, year & Animal & $\begin{array}{l}\text { Implant no, } \\
\text { dimensions } \\
\left(\mathrm{mm}^{2} \times \mathrm{mm}\right)\end{array}$ & Manufacturer & Material & $\begin{array}{l}\text { Surface } \\
\text { treatment }\end{array}$ & BIC (\%) & $\begin{array}{c}\mathrm{RT} \\
(\mathrm{N} / \mathrm{cm})\end{array}$ \\
\hline \multirow{6}{*}{$\begin{array}{l}\text { (Gahlert et al. } \\
\text { 2009) }\end{array}$} & \multirow{6}{*}{ Pigs } & \multirow{6}{*}{$4.1 \times 10$} & \multirow[t]{2}{*}{ Straumann AG } & \multirow[t]{2}{*}{$\mathrm{Zr}$} & \multirow[t]{3}{*}{$\begin{array}{l}\text { Hydrofluoric } \\
\text { acid }\end{array}$} & $\begin{array}{l}4 \text { weeks: } \\
27.1 \pm 3.5\end{array}$ & - \\
\hline & & & & & & $\begin{array}{l}8 \text { weeks: } \\
51.9 \pm 14\end{array}$ & - \\
\hline & & & \multirow{4}{*}{ Straumann AG } & \multirow{4}{*}{$\mathrm{Ti}$} & & $\begin{array}{l}12 \text { weeks: } \\
51.1 \pm 12.4\end{array}$ & - \\
\hline & & & & & \multirow[t]{3}{*}{ SLA } & $\begin{array}{l}4 \text { weeks: } \\
23.5 \pm 7.5\end{array}$ & - \\
\hline & & & & & & $\begin{array}{l}8 \text { weeks: } \\
53.3 \pm 27.6\end{array}$ & - \\
\hline & & & & & & $\begin{array}{l}12 \text { weeks: } \\
58.5 \pm 11.4\end{array}$ & - \\
\hline \multirow{6}{*}{$\begin{array}{l}\text { (Schliephake } \\
\text { et al. 2010) }\end{array}$} & \multirow{6}{*}{ Minipig } & 72 & SPI EIEMENT & $\mathrm{Zr}$ & Sandblasted & $\begin{array}{c}4 \text { weeks: } \\
57.5 \pm 14.3\end{array}$ & $\begin{array}{c}4 \text { weeks: } \\
55.9 \pm \\
18.4\end{array}$ \\
\hline & & \multirow[t]{5}{*}{$4.2 \times 8$} & & & & $\begin{array}{l}13 \text { weeks: } \\
54.6 \pm 17.6\end{array}$ & $\begin{array}{c}13 \\
\text { weeks: } \\
99.4 \pm \\
30.9\end{array}$ \\
\hline & & & - & $\mathrm{Zr}$ & $\begin{array}{c}\text { Sandblasted and } \\
\text { etched }\end{array}$ & $\begin{array}{l}4 \text { weeks: } \\
66.7 \pm 15.8\end{array}$ & $\begin{array}{c}4 \text { weeks: } \\
111.8 \pm \\
42.4\end{array}$ \\
\hline & & & \multirow{3}{*}{ - } & \multirow{3}{*}{$\mathrm{Ti}$} & \multirow{3}{*}{$\begin{array}{c}\text { Sandblasted and } \\
\text { etched }\end{array}$} & $\begin{array}{l}13 \text { weeks: } \\
57.6 \pm 23.7\end{array}$ & $\begin{array}{c}13 \\
\text { weeks: } \\
100.3 \pm \\
47\end{array}$ \\
\hline & & & & & & $\begin{array}{l}4 \text { weeks: } \\
69.3 \pm 17\end{array}$ & $\begin{array}{c}4 \text { weeks: } \\
244.5 \pm \\
34.9\end{array}$ \\
\hline & & & & & & $\begin{array}{l}13 \text { weeks: } \\
78.9 \pm 5.8\end{array}$ & $\begin{array}{c}13 \\
\text { weeks: } \\
221.9 \pm \\
27.1\end{array}$ \\
\hline \multirow{6}{*}{$\begin{array}{l}\text { (Depprich et } \\
\text { al. 2008) }\end{array}$} & \multirow{6}{*}{ Minipig } & 48 & - & $\mathrm{Zr}$ & Acid etched & $\begin{array}{c}1 \text { week; } \\
35.3 \pm 10.8\end{array}$ & - \\
\hline & & $3.5 \times 9$ & & & & $\begin{array}{l}4 \text { weeks: } \\
45.3 \pm 15.7\end{array}$ & - \\
\hline & & & & & & $\begin{array}{l}12 \text { weeks: } \\
71.4 \pm 17.8\end{array}$ & - \\
\hline & & & - & $\mathrm{Ti}$ & Acid etched & $\begin{array}{c}1 \text { week; } \\
47.7 \pm 9.1\end{array}$ & - \\
\hline & & & & & & $\begin{array}{c}4 \text { weeks: } \\
58.6 \pm 9.5\end{array}$ & - \\
\hline & & & & & & $\begin{array}{l}12 \text { weeks: } \\
82.9 \pm 10.7\end{array}$ & - \\
\hline \multirow{3}{*}{$\begin{array}{l}\text { Kohal et al. } \\
2016\end{array}$} & \multirow{3}{*}{ Monkey } & 24 & & & & 9 months: & \\
\hline & & $4 \times 13$ & - & $\mathrm{Ti}$ & SLA & $72.9 \pm 14$ & - \\
\hline & & $4 \times 15$ & - & $\mathrm{Zr}$ & Sandblasted & $67.4 \pm 17$ & - \\
\hline
\end{tabular}




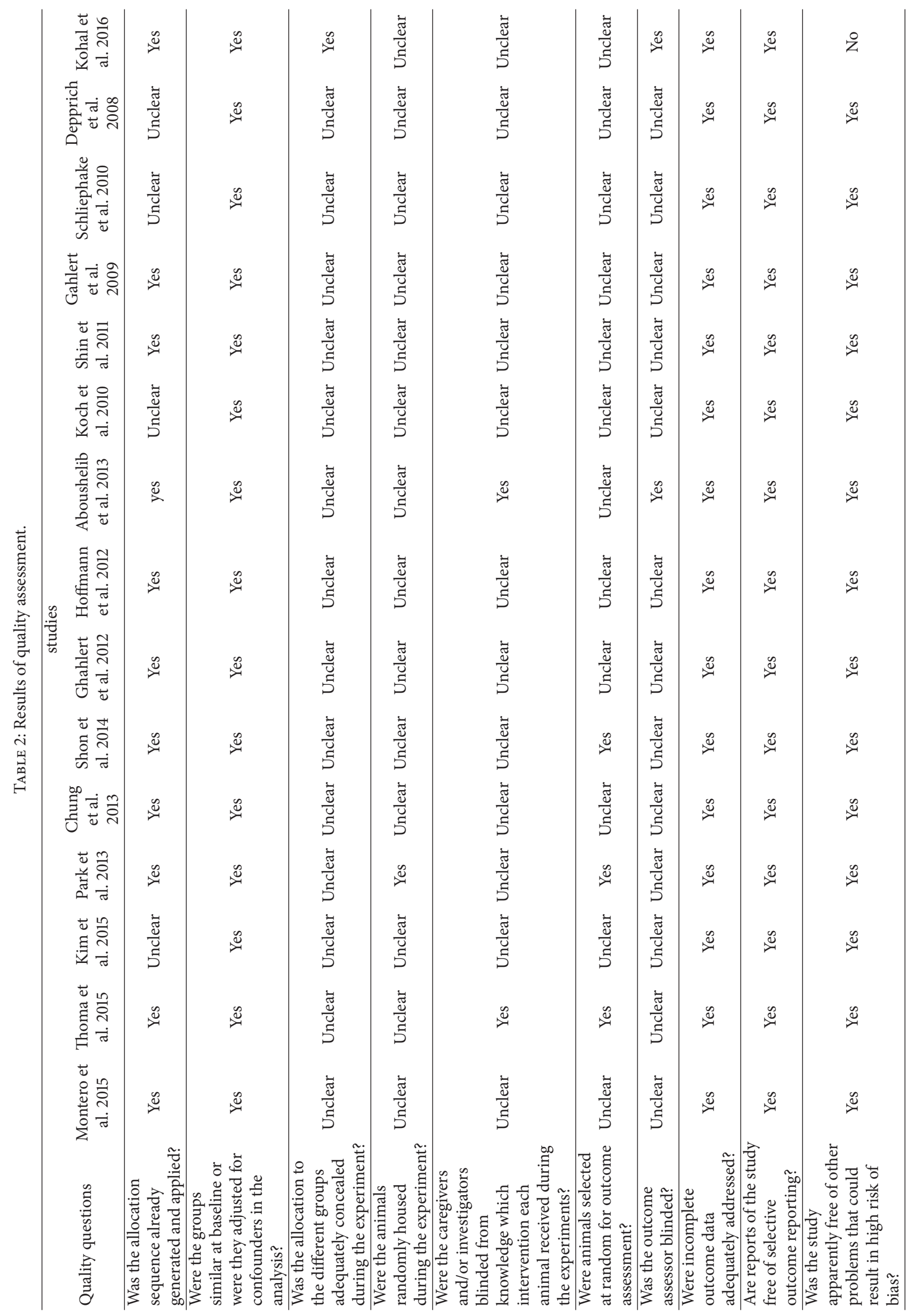




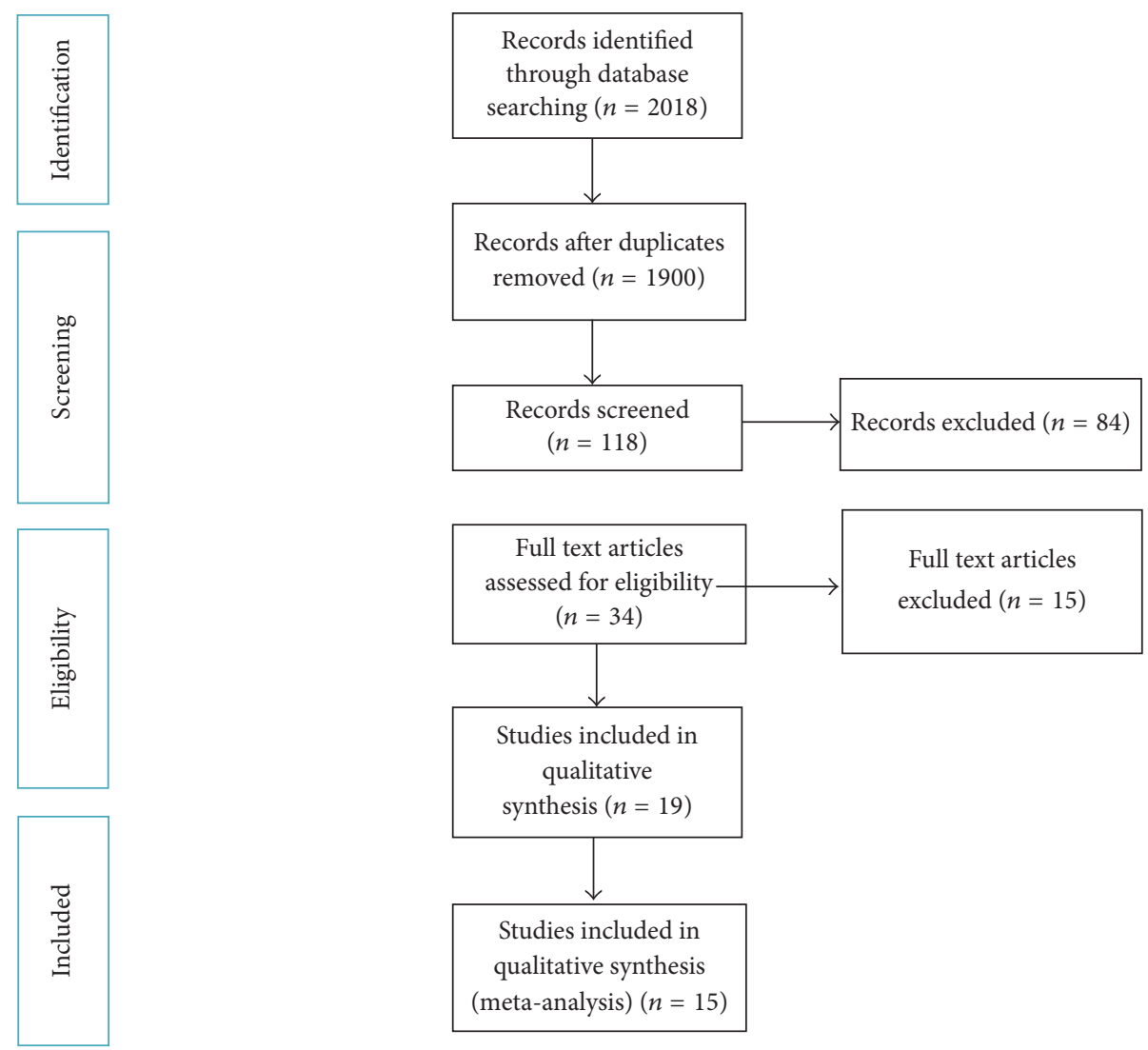

FIGURE 1: Diagram of the search strategy according to PRISMA statement.

implants were installed in 4 studies [15, 27-29]. Five studies compared RT values of zirconia with modified zirconia implants [14, 15, 27-29].

3.2.1. Bone to Implant Contact (BIC). Fifteen studies assessed the mean BIC values (\%) around the implants in different observation periods. The range of BIC values for titanium, machined zirconia, blasted zirconia, and surface etched zirconia implant groups in selected studies was $31.80 \%$ to $87.85 \%, 33.74 \%$ to $84.17 \%, 41.35 \%$ to $67.4 \%$, and $51.1 \%$ to $71.4 \%$, respectively.

The results of the studies suggested no significant difference in the BIC values between titanium and machined zirconia (standard difference in mean: $0.138,95 \% \mathrm{CI}$ : -0.166 to $0.443 ; p=0.373$ ) and between titanium and blasted zirconia implants (standard difference in mean: 0.041, 95\% CI: -0.407 to $0.488 ; p=0.859$ ). However, a significant increase was observed in BIC values of acid etched zirconia compared with titanium implants (standard difference in mean: $0.766,95 \%$ CI: 0.068 to $1.461 ; p=0.032$ ). Comparative studies of machined and surfaced modified zirconia implants showed a greater BIC for unmodified zirconia implants (standard difference in mean: $-0.526,95 \% \mathrm{CI}:-0.973$ to $-0.080 ; p=0.021)$. In addition, PIM untreated zirconia implants showed significantly greater BIC than PIM-treated zirconia implants (standard difference in mean: $-0.622,95 \%$ CI: -1.012 to $-0.232 ; p=0.002$ ) (Figure 2 ).
3.2.2. Removal Torque (RT). All five accepted studies showed more favorable RT values for untreated zirconia implants than those of machined zirconia implants, which were statistically significant (standard difference in mean: $-0.749,95 \%$ CI: -1.157 to $-0.341 ; p<0.001$ ) (Figure 3 ).

\section{Discussion}

This systematic review and meta-analysis focused on 2 questions. (1) Does the choice of the implant material affect BIC when comparing titanium to zirconia in animal models? (2) What is the impact of zirconia implant surface modification on BIC and RT when comparing modified to as sintered zirconia in animal models? Data synthesis showed a significantly better BIC values for acid etched and unmodified zirconia implants compared with those of titanium and modified-surface zirconia implants respectively. Thus, the null hypotheses were rejected.

It is well known that surface topography, chemistry, and roughness affect the rate and quality of new tissue formation [30]. Surface modification can enhance bone healing and integration of titanium implants and result in higher bone-implant contact ratios [20, 31]; however, the interfacial interaction of modified zirconia with bone is still not fully understood. A number of animal studies have been published that outline hard and soft tissue integration of zirconia implants on the histological level and have shown 


\begin{tabular}{|c|c|c|c|c|c|c|c|c|c|c|c|c|c|c|c|}
\hline \multirow{2}{*}{ Study name } & \multicolumn{4}{|c|}{ Sample size } & \multicolumn{4}{|c|}{ Statistics for each study } & \multicolumn{7}{|c|}{ Std diff in means and 95\% CI } \\
\hline & $\begin{array}{c}\text { Unm } \\
\mathrm{Ti}\end{array}$ & $\begin{array}{l}\text { dified } \\
\mathrm{Zr}\end{array}$ & $\begin{array}{l}\text { Std diff } \\
\text { in means }\end{array}$ & $\begin{array}{l}\text { Standard } \\
\text { error }\end{array}$ & Variance & $\begin{array}{c}\text { Lower } \\
\text { limit }\end{array}$ & $\begin{array}{c}\text { Upper } \\
\text { limit }\end{array}$ & $Z$-value & $p$ value & & & & & & $\begin{array}{r}\text { Relative } \\
\text { weight }\end{array}$ \\
\hline Kohal et al. 2016 & 7 & 7 & 1.082 & 0.572 & 0.328 & -0.039 & 2.204 & 1.891 & 0.059 & & & & & $\rightarrow$ & 7.37 \\
\hline Thoma et al. 2015 & 12 & 12 & 0.183 & 0.409 & 0.167 & -0.619 & 0.984 & 0.446 & 0.656 & & & & & & 14.42 \\
\hline Koch et al. 2010 & 12 & 12 & -2.050 & 0.504 & 0.254 & -3.038 & -1.061 & -4.065 & 0.000 & $k$ & & & & & 9.49 \\
\hline Hoffmann et al. 2012 & 24 & 24 & 0.080 & 0.289 & 0.083 & -0.486 & 0.646 & 0.277 & 0.782 & & & & & & 28.93 \\
\hline Shin et al. 2011 & 10 & 10 & 0.499 & 0.454 & 0.206 & -0.391 & 1.389 & 1.099 & 0.272 & & & & & & 11.70 \\
\hline \multirow{3}{*}{ Montero et al. 2015} & 91 & 91 & 0.138 & 0.155 & 0.024 & -0.166 & 0.443 & 0.890 & 0.373 & & & & & & \\
\hline & & & & & & & & & & -1.00 & -0.50 & 0.00 & 0.50 & 1.00 & \\
\hline & & & & & & & & & & & Favors A & & Favors B & & \\
\hline
\end{tabular}

Meta-analysis

(a)

\begin{tabular}{|c|c|c|c|c|c|c|c|c|c|c|c|c|c|c|}
\hline \multirow[t]{2}{*}{ Study name } & \multicolumn{3}{|c|}{ Sample size } & \multicolumn{5}{|c|}{ Statistics for each study } & \multicolumn{6}{|c|}{ Std diff in means and 95\% CI } \\
\hline & $\mathrm{Ti}$ & $\begin{array}{c}\text { Zr- } \\
\text { blasted }\end{array}$ & $\begin{array}{l}\text { Std diff } \\
\text { in means }\end{array}$ & $\begin{array}{l}\text { Standard } \\
\text { error }\end{array}$ & Variance & $\begin{array}{c}\text { Lower } \\
\text { limit }\end{array}$ & $\begin{array}{c}\text { Upper } \\
\text { limit }\end{array}$ & $Z$-value & $p$ value & & & & & $\begin{array}{r}\text { Relative } \\
\text { weight }\end{array}$ \\
\hline Hoffman et al. 2012 & 24 & 22 & -0.465 & 0.299 & 0.089 & -1.051 & 0.121 & -1.555 & 0.120 & & + & & & 58.26 \\
\hline Schliephake et al. 2010 & 6 & 6 & 1.854 & 0.690 & 0.477 & 0.501 & 3.208 & 2.686 & 0.007 & & & & & 10.94 \\
\hline \multirow[t]{4}{*}{ Kohal et al. 2016} & 12 & 12 & 0.353 & 0.411 & 0.169 & -0.453 & 1.160 & 0.858 & 0.391 & & & & & 30.80 \\
\hline & 42 & 40 & 0.041 & 0.228 & 0.052 & -0.407 & 70.488 & 0.178 & 0.859 & & & & & \\
\hline & & & & & & & & & -1.00 & -0.50 & 0.00 & 0.50 & 1.00 & \\
\hline & & & & & & & & & & Favors A & & Favors B & & \\
\hline
\end{tabular}

Meta-analysis

(b)

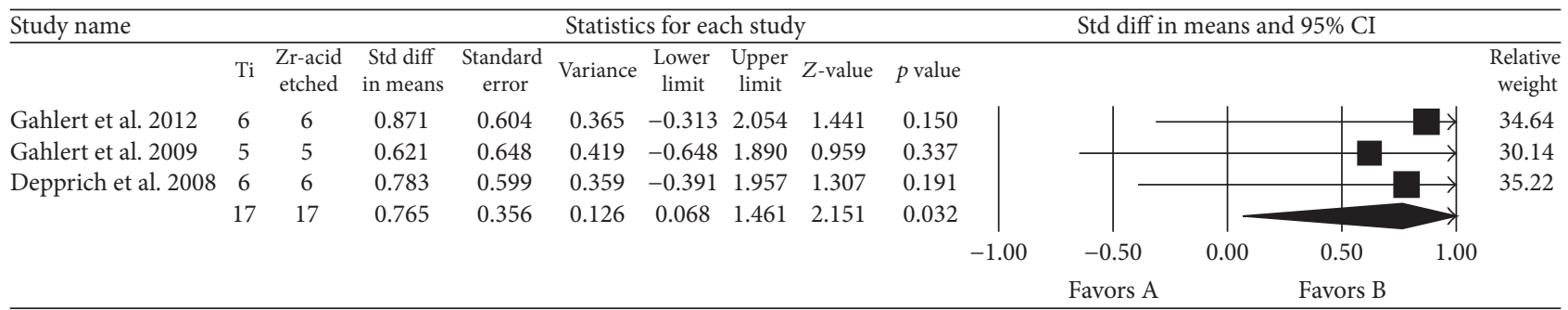

Meta-analysis

(c)

\begin{tabular}{|c|c|c|c|c|c|c|c|c|c|c|c|c|c|c|c|}
\hline \multirow[t]{2}{*}{ Study name } & \multicolumn{3}{|c|}{ Sample size } & \multicolumn{5}{|c|}{ Statistics for each study } & \multicolumn{7}{|c|}{ Std diff in means and 95\% CI } \\
\hline & $\mathrm{Zr}$ & $\begin{array}{c}\text { Modified } \\
\mathrm{Zr}\end{array}$ & $\begin{array}{l}\text { Std diff } \\
\text { in means }\end{array}$ & $\begin{array}{c}\text { Standard } \\
\text { error }\end{array}$ & Variance & $\begin{array}{c}\text { Lower } \\
\text { limit }\end{array}$ & $\begin{array}{l}\text { Upper } \\
\text { limit }\end{array}$ & $Z$-value & $p$ value & & & & & & $\begin{array}{r}\text { Relative } \\
\text { weight }\end{array}$ \\
\hline Thoma et al. 2015 & 12 & 12 & 0.424 & 0.413 & 0.170 & -0.385 & 1.233 & 1.028 & 0.304 & & & & 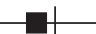 & & 30.49 \\
\hline Hoffmann et al. 2012 & 24 & 22 & -0.502 & 0.300 & 0.090 & -1.089 & 0.086 & -1.674 & 0.094 & & & & & & 57.82 \\
\hline \multirow[t]{4}{*}{ Aboushelib et al. 2013} & 10 & 10 & -3.128 & 0.667 & 0.445 & -4.435 & -1.821 & -4.691 & 0.000 & & & & & & 11.69 \\
\hline & 46 & 44 & -0.526 & 0.228 & 0.052 & -0.973 & -0.080 & -2.309 & 0.021 & & & & & & \\
\hline & & & & & & & & & & -1.00 & -0.50 & 0.00 & 0.50 & 1.00 & \\
\hline & & & & & & & & & & & Favo & & Favors $\mathrm{F}$ & & \\
\hline
\end{tabular}

Meta-analysis

(d)

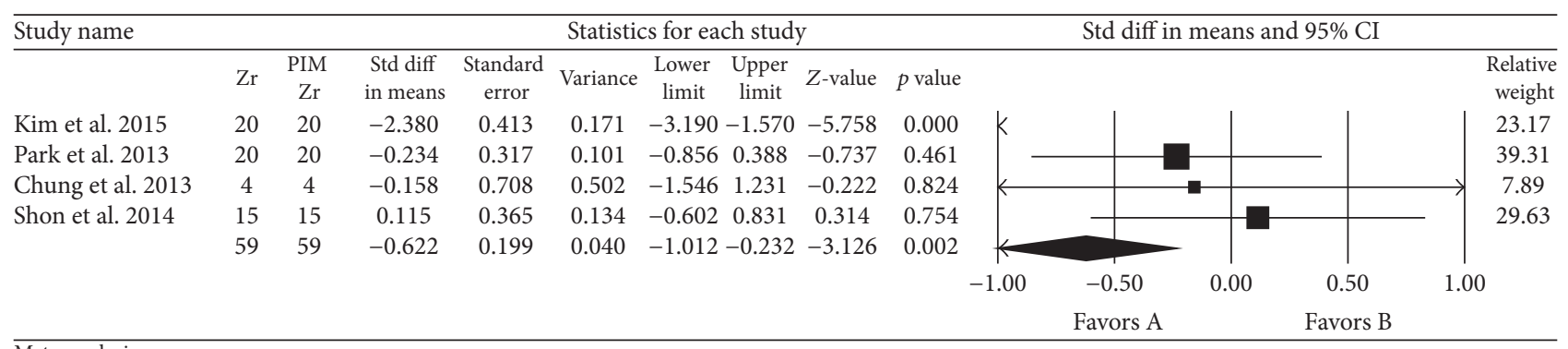

Meta-analysis

(e)

FIGURE 2: Forest plot for the event "BIC" in the comparison between titanium and zirconia implants (a), between titanium and sandblasted zirconia (b), between titanium and acid etched zirconia (c), between surface-modified and machined zirconia implants (d), and between untreated and PIM roughened mold zirconia implants (e). 


\begin{tabular}{|c|c|c|c|c|c|c|c|c|c|c|c|c|}
\hline \multirow[t]{2}{*}{ Study name } & \multicolumn{3}{|c|}{ Sample size } & \multicolumn{4}{|c|}{ Statistics for each study } & \multicolumn{5}{|c|}{ Std diff in means and 95\% CI } \\
\hline & $\mathrm{Zr}$ & $\begin{array}{c}\mathrm{Zr}- \\
\text { modified }\end{array}$ & $\begin{array}{c}\text { Std diff } \\
\text { in means }\end{array}$ & $\begin{array}{c}\text { Standard } \\
\text { error }\end{array}$ & Variance & $\begin{array}{cc}\text { Lower } & \text { Upper } \\
\text { limit } & \text { limit }\end{array}$ & $Z$-value & $p$ value & & & & $\begin{array}{r}\text { Relative } \\
\text { weight }\end{array}$ \\
\hline Kim et al. 2015 & 11 & 12 & -3.874 & 0.707 & 0.500 & $-5.260-2.487$ & -5.476 & $0.000 k$ & & & & 8.66 \\
\hline Park et al. 2013 & 7 & 7 & -2.116 & 0.668 & 0.446 & $-3.424-0.807$ & -3.170 & $0.002 k$ & & & & 9.72 \\
\hline Chung et al. 2013 & 10 & 10 & -1.681 & 0.520 & 0.271 & $-2.701-0.661$ & -3.231 & 0.001 & & & & 16.01 \\
\hline Shon et al. 2014 & 10 & 10 & -1.623 & 0.516 & 0.266 & $-2.633-0.612$ & -3.147 & 0.002 & & & & 16.30 \\
\hline \multirow[t]{3}{*}{ Hoffmann et al. 2012} & 24 & 24 & 0.661 & 0.296 & 0.088 & $0.080 \quad 1.242$ & 2.230 & 0.026 & & & & 49.30 \\
\hline & & & & & & & & -1.00 & -0.50 & 0.00 & 0.50 & 1.00 \\
\hline & & & & & & & & & Favors A & & Favors & \\
\hline
\end{tabular}

Meta-analysis

FIGURE 3: Forest plot for the event "RT" in the comparison between surface-modified and unmodified zirconia implants.

promising results $[29,32,33]$. Further, cell culture studies have demonstrated that zirconia especially with a moderately rough surface is accepted by osteoblasts and integrates into bone tissue $[9,34]$. Scarano et al. [35] examined the boneimplant interface of machined zirconia implants at 4 weeks of healing and reported BIC values of $68.4 \%$. Similarly, Akagawa et al. [36] observed a BIC ratio of $66 \%$ to $81 \%$ for zirconia implants inserted into mandible of monkeys after 24 months of healing which was similar to that of zirconia implants at the 12-month observation. It is noteworthy to mention that the current review included only those studies comparing unmodified or modified zirconia with titanium implants in order to perform a direct comparison. The present meta-analysis found an equivalent BIC values for machined zirconia compared with titanium implants. The lack of significant difference between the zirconia and titanium implants could lead to the conclusion that the zirconia implant is as osteoconductive as the titanium implant. For titanium implants, roughened surfaces have demonstrated superiority compared to their smooth, machined predecessors $[7,9]$. Consequently, zirconia implants with a roughened surface have been suggested to be capable of achieving greater stability in bone than machined zirconia implants [29]. Gahlert et al. confirmed that the increased surface roughness of sandblasted and acid etched zirconia implants not only has an important influence on bone integration but also is associated with increased removal torque strength and bone stability in minipigs [6]. Zirconia implants with rough surfaces have also demonstrated higher removal torque resistance in rabbits [18]. The osseointegration capacity of machined zirconia surface is substantially increased after modification by sandblasting [20]. However, Gahlert et al. [6] specified that further improvements in the surface roughness of zirconia implants are needed. As the review only included animal studies, the analysis of each surface modification was hindered by the limited number of studies, and only the two surface modifications (acid etching or blasting) were compared with titanium implants. According to the meta-analysis, no significant difference was found in the BIC values between titanium and blasted zirconia implants; however, the surface modification for the acid etched zirconia surfaces resulted in significantly higher BIC compared with the titanium implants. The evaluated histological data were commonly in agreement with this result in which the modified zirconia implants were comparable to titanium implants. However, Gahlert et al. [6] and Depprich et al. [24] demonstrated that the BIC values obtained for the entire implant thread length had no significant difference between modified zirconia and titanium implants in minipig models during the observation periods. Thus, the authors concluded that there is an evidence for a better outcome of surfacemodified zirconia implants over titanium implants.

When comparing the BIC values of modified zirconia surfaces relative to the machined ones, data synthesis has identified that unmodified zirconia surface may be favored over modified zirconia implant. This result was supported by the RT values which also favored to zirconia implants. When evaluating the outcomes reported in these studies, it must be emphasized that mean BIC values assessed after respective healing periods were commonly in agreement with this result. Accordingly, the bone to implant contact around zirconia implants exhibited greater values than those of the modified zirconia implants [14, 21]. Conversely, Aboushelib et al. [18] reported greater bone to implant contact for selective infiltration etched zirconia implants after healing periods $(65.38 \pm 5.7 \%$ at 4 weeks and $75.01 \pm 5.1 \%$ at 6 weeks $)$, confirming a better bone reaction to modified zirconia. However, it is not considered correct to present comparative data without defining surface roughness. Even a machined zirconia surface may vary considerably in roughness as is the case for blasted, acid etched, or other modified surfaces. Thus different results may be reported of the same surface topography $[37,38]$. Unfortunately, detailed information on surface topography of all included studies was not given. This makes it increasingly difficult to compare different study outcomes particularly when the techniques used for surface modifications vary considerably. Also, the manufacturing technique and the chemical and physical composition of zirconia implants show substantial differences [39]. Hence, a surface that is termed rough in one study may be termed smooth in another. In addition, tissue response to altered surface topography need not necessarily reflect the performed change of the surface alone. When the surface topography is changed, the surface chemistry or physics may change simultaneously [40]. These factors seem to play important roles in the osseointegration of modified zirconia implants, although it is not yet clear which are most important. So although tendencies for improved osseointegration following 
zirconia implant modification were seen, further researches and experiments should focus on these materials.

It can be concluded that an acceptable BIC is achieved after the healing period independent of implant material and surface treatment. There is a general consensus that roughening the implant surface above the level seen with most machined surfaces enhances the bone response to implants. However, the effects of surface roughness on the cell function and interplay between a zirconia implant and the adjoining bone tissue have to be clearly investigated.

Some of the challenges in the translation of animal studies are decreased by meta-analysis of animal studies; however, the results have to be interpreted with caution because of the presence of several confounding factors. The available literature on bone-implant interface involves a wide range of studies with various methods and results; thus the impact of one surface over another can barely be compared [41]. Arguably, the most insidious source of confounding is the method of bone to implant contact assessment. By far, histomorphometric analysis has been the gold standard in evaluating the bone-implant interface. The various methods of the histomorphometric analysis, microscopic magnification, and different software and algorithms appear to play a crucial role in BIC calculations which may subsequently lead to deviant results $[29,32]$. The bone tissue has to be transferred to specialized laboratory for preparation immediately after animal sacrifice. Besides the various methods of fixation used in research protocols, preparation of thin tissue and fixation of animal tissue in formalin may disturb the results. Moreover, variation in the area to be evaluated or region of interest exists among the studies. Obviously, the values obtained from the entire implant surface may characterize the performance in the clinically realistic context [25]. However, different regions of interest like the whole implant surface, the mineralized zone between two threads, and the contact at the best three threads were reported in evaluated studies.

Another important issue to consider is the animals used for the experiment. Pigs and rabbit were commonly used as experimental models in accepted studies, while monkey and rat were used in two studies. Variation in animal models among studies may result in considerable variations in the results [32]. Selection of an appropriate animal model for demonstrating the response of bone tissue to biomaterial is difficult, mainly because the bone characteristics, microstructure, composition, modeling, and remodeling are different from those of humans [40]. While dogs, sheep, goats, pigs, or rabbits are suitable models for evaluating implants (according to international standards), no species fulfils the requirements of an ideal model. It is important to consider the research question when selecting the animal model. The characteristics of human bone are best approximated by dog models; but substantial differences exist in the bone anatomy, microstructure, and remodeling between rabbit and humane [42]. Rat is unsuitable due to significant dissimilarities in bone structure [43].

Considering these limitations, the findings of the current study should be interpreted cautiously. Several other confounding factors such as surface topography, chemistry, roughness, implant design and dimension, and healing time influence the results. Coexistence of multiple factors in the studies makes the evaluation of one particular factor impossible and the lack of control over these factors lowers the potential of definitive result extraction.

\section{Conclusion}

Within the limitation of the present study, the following conclusions can be drawn.

(1) No significant difference in the BIC values was observed between titanium and zirconia and between titanium and sandblasted zirconia implants, (2) a significantly better BIC was observed for surface treated zirconia compared with titanium implants, (3) unmodified zirconia implants showed favorable BIC values compared to machined zirconia implants; moreover, PIM untreated zirconia implants showed significantly better results than PIM-treated zirconia implants, and (4) untreated zirconia implants showed favorable RT values compared to machined zirconia implants.

\section{Competing Interests}

The authors declare that there is no conflict of interests regarding the publication of this paper.

\section{References}

[1] M. Hisbergues, S. Vendeville, and P. Vendeville, "Review zirconia: established facts and perspectives for a biomaterial in dental implantology," Journal of Biomedical Materials Research-Part B Applied Biomaterials, vol. 88, no. 2, pp. 519-529, 2009.

[2] Z. Özkurt and E. Kazazoğlu, "Zirconia dental implants: a literature review," Journal of Oral Implantology, vol. 37, no. 3, pp. 367-376, 2011.

[3] G. P. Jayaswal, S. P. Dange, and A. N. Khalikar, "Bioceramic in dental implants: a review," Journal of Indian Prosthodontist Society, vol. 10, no. 1, pp. 8-12, 2010.

[4] K. M. Regish, D. Sharma, and D. R. Prithviraj, "An overview of immediate root analogue zirconia implants," Journal of Oral Implantology, vol. 39, no. 2, pp. 225-233, 2013.

[5] S. Parithimarkalaignan and T. V. Padmanabhan, "Osseointegration: an update," Journal of Indian Prosthodontist Society, vol. 13, no. 1, pp. 2-6, 2013.

[6] M. Gahlert, S. Roehling, C. M. Sprecher, H. Kniha, S. Milz, and K. Bormann, "In vivo performance of zirconia and titanium implants: a histomorphometric study in mini pig maxillae," Clinical Oral Implants Research, vol. 23, no. 3, pp. 281-286, 2012.

[7] T. Albrektsson, P.-I. Brånemark, H.-A. Hansson, and J. Lindström, "Osseointegrated titanium implants: requirements for ensuring a long-lasting, direct bone-to-implant anchorage in man," Acta Orthopaedica, vol. 52, no. 2, pp. 155-170, 1981.

[8] S. Bauer, P. Schmuki, K. von der Mark, and J. Park, "Engineering biocompatible implant surfaces. Part I: materials and surfaces," Progress in Materials Science, vol. 58, no. 3, pp. 261-326, 2013.

[9] T. Albrektsson and A. Wennerberg, "Oral implant surfaces: part 1-review focusing on topographic and chemical properties of different surfaces and in vivo responses to them," International Journal of Prosthodontics, vol. 17, no. 5, pp. 536-543, 2004. 
[10] L. Sennerby, A. Dasmah, B. Larsson, and M. Iverhed, "Bone tissue responses to surface-modified zirconia implants: a histomorphometric and removal torque study in the rabbit," Clinical Implant Dentistry and Related Research, vol. 7, supplement 1, pp. S13-S20, 2005.

[11] B. Bacchelli, G. Giavaresi, M. Franchi et al., "Influence of a zirconia sandblasting treated surface on peri-implant bone healing: an experimental study in sheep," Acta Biomaterialia, vol. 5, no. 6, pp. 2246-2257, 2009.

[12] G. Schierano, F. Mussano, M. G. Faga et al., "An alumina toughened zirconia composite for dental implant application: in vivo animal results," BioMed Research International, vol. 2015, Article ID 157360, 9 pages, 2015.

[13] J. Lee, J. H. Sieweke, N. A. Rodriguez et al., "Evaluation of nanotechnology-modified zirconia oral implants: a study in rabbits," Journal of Clinical Periodontology, vol. 36, no. 7, pp. 610-617, 2009.

[14] O. Hoffmann, N. Angelov, G.-G. Zafiropoulos, and S. Andreana, "Osseointegration of zirconia implants with different surface characteristics: an evaluation in rabbits," The International Journal of Oral \& Maxillofacial Implants, vol. 27, no. 2, pp. 352358, 2012.

[15] H.-K. Kim, K. M. Woo, W.-J. Shon, J.-S. Ahn, S. Cha, and Y.S. Park, "Comparison of peri-implant bone formation around injection-molded and machined surface zirconia implants in rabbit tibiae," Dental Materials Journal, vol. 34, no. 4, pp. 508$515,2015$.

[16] D. Moher, A. Liberati, J. Tetzlaff, and D. G. Altman, "Reprintpreferred reporting items for systematic reviews and metaanalyses: the PRISMA statement," Physical Therapy, vol. 89, no. 9, pp. 873-880, 2009.

[17] K.-Y. Chen, B. Ma, Y.-N. Wang et al., "SYRCLE's risk of bias tool for animal studies," Chinese Journal of Evidence-Based Medicine, vol. 14, no. 10, pp. 1281-1285, 2014.

[18] M. N. Aboushelib, N. A. Salem, A. L. A. Taleb, and N. M. A. E. Moniem, "Influence of surface nano-roughness on osseointegration of zirconia implants in rabbit femur heads using selective infiltration etching technique," Journal of Oral Implantology, vol. 39, no. 5, pp. 583-590, 2013.

[19] F. P. Koch, D. Weng, S. Krämer, S. Biesterfeld, A. JahnEimermacher, and W. Wagner, "Osseointegration of one-Piece zirconia implants compared with a titanium implant of identical design: a histomorphometric study in the dog," Clinical Oral Implants Research, vol. 21, no. 3, pp. 350-356, 2010.

[20] R. J. Kohal, M. Bächle, A. Renz, and F. Butz, "Evaluation of alumina toughened zirconia implants with a sintered, moderately rough surface: an experiment in the rat," Dental Materials, vol. 32, no. 1, pp. 65-72, 2016.

[21] D. S. Thoma, G. I. Benic, F. Muñoz et al., "Histological analysis of loaded zirconia and titanium dental implants: an experimental study in the dog mandible," Journal of Clinical Periodontology, vol. 42, no. 10, pp. 967-975, 2015.

[22] D. Shin, S. B. Blanchard, M. Ito, and T.-M. G. Chu, "Peripheral quantitative computer tomographic, histomorphometric, and removal torque analyses of two different non-coated implants in a rabbit model," Clinical Oral Implants Research, vol. 22, no. 3, pp. 242-250, 2011.

[23] J. Montero, M. Bravo, Y. Guadilla et al., "Comparison of clinical and histologic outcomes of zirconia versus titanium implants placed in fresh sockets: a 5-month study in beagles," The International Journal of Oral \& Maxillofacial Implants, vol. 30, no. 4, pp. 773-780, 2015.
[24] R. Depprich, H. Zipprich, M. Ommerborn et al., “Osseointegration of zirconia implants compared with titanium: an in vivo study," Head and Face Medicine, vol. 4, no. 1, article no. 30, 2008.

[25] M. Gahlert, S. Röhling, M. Wieland, C. M. Sprecher, H. Kniha, and S. Milz, "Osseointegration of zirconia and titanium dental implants: a histological and histomorphometrical study in the maxilla of pigs," Clinical Oral Implants Research, vol. 20, no. 11, pp. 1247-1253, 2009.

[26] H. Schliephake, T. Hefti, F. Schlottig, P. Gédet, and H. Staedt, "Mechanical anchorage and peri-implant bone formation of surface-modified zirconia in minipigs," Journal of Clinical Periodontology, vol. 37, no. 9, pp. 818-828, 2010.

[27] W.-J. Shon, S. H. Chung, H.-K. Kim, G.-J. Han, B.-H. Cho, and Y.-S. Park, "Peri-implant bone formation of non-thermal atmospheric pressure plasma-treated zirconia implants with different surface roughness in rabbit tibiae," Clinical Oral Implants Research, vol. 25, no. 5, pp. 573-579, 2014.

[28] S. H. Chung, H.-K. Kim, W.-J. Shon, and Y.-S. Park, "Periimplant bone formations around $(\mathrm{Ti}, \mathrm{Zr}) \mathrm{O}_{2}$-coated zirconia implants with different surface roughness," Journal of Clinical Periodontology, vol. 40, no. 4, pp. 404-411, 2013.

[29] Y.-S. Park, S.-H. Chung, and W.-J. Shon, "Peri-implant bone formation and surface characteristics of rough surface zirconia implants manufactured by powder injection molding technique in rabbit tibiae," Clinical Oral Implants Research, vol. 24, no. 5, pp. 586-591, 2013.

[30] D. D. Deligianni, N. Katsala, S. Ladas, D. Sotiropoulou, J. Amedee, and Y. F. Missirlis, "Effect of surface roughness of the titanium alloy Ti-6Al-4V on human bone marrow cell response and on protein adsorption," Biomaterials, vol. 22, no. 11, pp. 1241-1251, 2001.

[31] K. M. Hotchkiss, N. B. Ayad, S. L. Hyzy, B. D. Boyan, and R. Olivares-Navarrete, "Dental implant surface chemistry and energy alter macrophage activation in vitro," Clinical Oral Implants Research, 2016.

[32] T. Gredes, P. Kubasiewicz-Ross, T. Gedrange, M. Dominiak, and C. Kunert-Keil, "Comparison of surface modified zirconia implants with commercially available zirconium and titanium implants: a histological study in pigs," Implant Dentistry, vol. 23, no. 4, pp. 502-507, 2014.

[33] A. Liñares, L. Grize, F. Muñoz et al., "Histological assessment of hard and soft tissues surrounding a novel ceramic implant: a pilot study in the minipig," Journal of Clinical Periodontology, vol. 43 , no. 6, pp. 538-546, 2016.

[34] T. Hirano, H. Sasaki, S. Honma et al., "Proliferation and osteogenic differentiation of human mesenchymal stem cells on zirconia and titanium with different surface topography," Dental Materials Journal, vol. 34, no. 6, pp. 872-880, 2015.

[35] A. Scarano, F. Di Carlo, M. Quaranta, and A. Piattelli, "Bone response to zirconia ceramic implants: an experimental study in rabbits," The Journal of Oral Implantology, vol. 29, no. 1, pp. 8-12, 2003.

[36] Y. Akagawa, R. Hosokawa, Y. Sato, and K. Kamayama, "Comparison between freestanding and tooth-connected partially stabilized zirconia implants after two years' function in monkeys: a clinical and histologic study," Journal of Prosthetic Dentistry, vol. 80 , no. 5 , pp. $551-558,1998$.

[37] M. Wieland, M. Textor, N. D. Spencer, and D. M. Brunette, "Wavelength-dependent roughness: a quantitative approach to characterizing the topography of rough titanium surfaces," International Journal of Oral and Maxillofacial Implants, vol. 16, no. 2, pp. 163-181, 2001. 
[38] A. Wennerberg, T. Albrektsson, and J. Lausmaa, “Torque and histomorphometric evaluation of c.p. titanium screws blasted with 25- and 75- $\mu \mathrm{m}$-sized particles of Al2O3," Journal of Biomedical Materials Research, vol. 30, no. 2, pp. 251-260, 1996.

[39] S. Zinelis, A. Thomas, K. Syres, N. Silikas, and G. Eliades, "Surface characterization of zirconia dental implants," Dental Materials, vol. 26, no. 4, pp. 295-305, 2010.

[40] J. D. Langhoff, K. Voelter, D. Scharnweber et al., "Comparison of chemically and pharmaceutically modified titanium and zirconia implant surfaces in dentistry: a study in sheep," International Journal of Oral and Maxillofacial Surgery, vol. 37, no. 12, pp. 1125-1132, 2008.

[41] E. Sagırkaya, A. S. Kucukekenci, D. Karasoy, K. Akça, S. E. Eckert, and M. C. Çehreli, "Comparative assessments, metaanalysis, and recommended guidelines for reporting studies on histomorphometric bone-implant contact in humans," The International Journal of Oral \& Maxillofacial Implants, vol. 28, no. 5, pp. 1243-1253, 2013.

[42] A. I. Pearce, R. G. Richards, S. Milz, E. Schneider, and S. G. Pearce, "Animal models for implant biomaterial research in bone: a review," European Cells and Materials, vol. 13, pp. 1-10, 2007.

[43] A. S. Turner, "Animal models of osteoporosis-necessity and limitations," European Cells and Materials, vol. 1, pp. 66-81, 2001. 

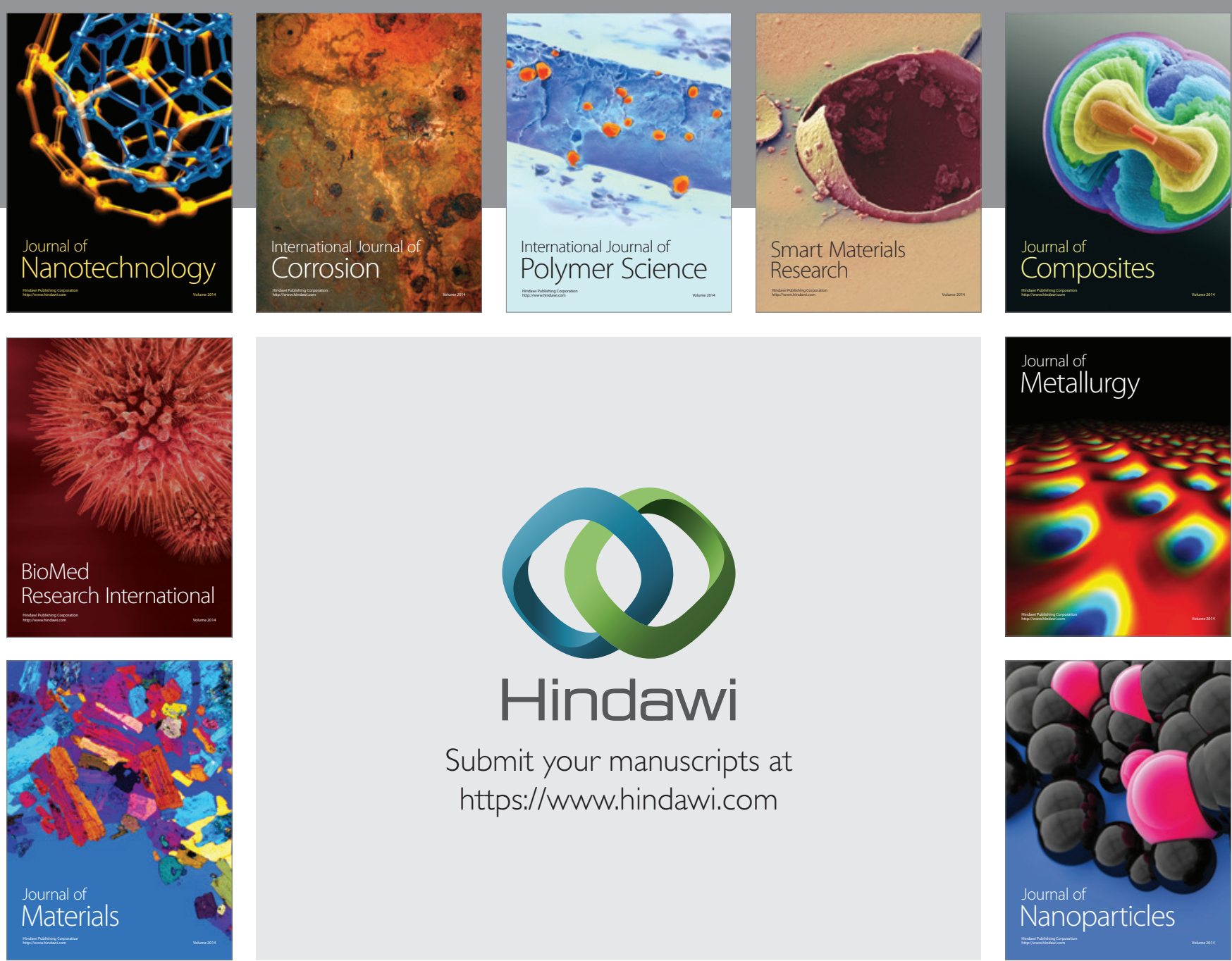

\section{Hindawi}

Submit your manuscripts at

https://www.hindawi.com

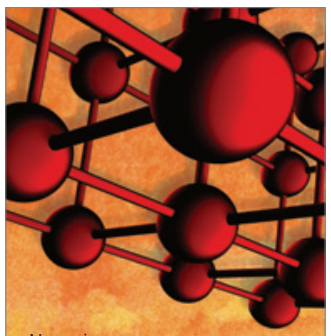

Materials Science and Engineering
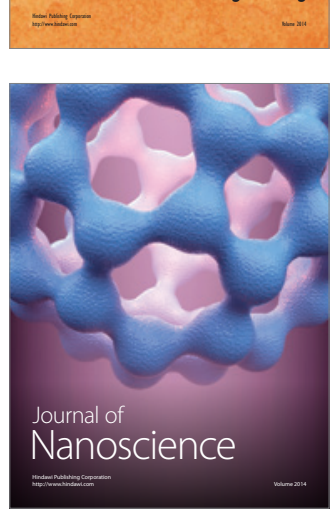
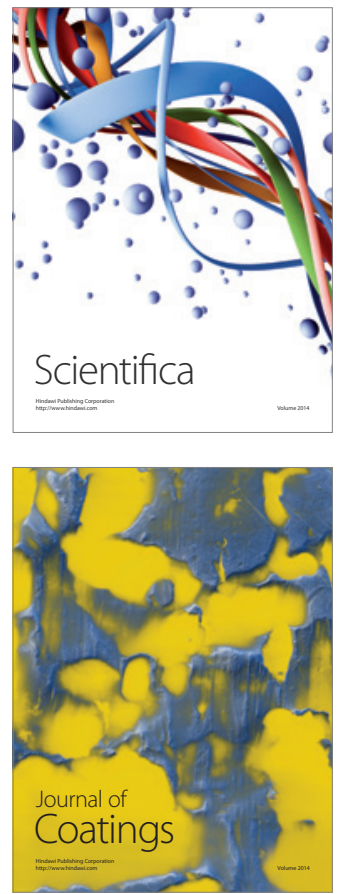
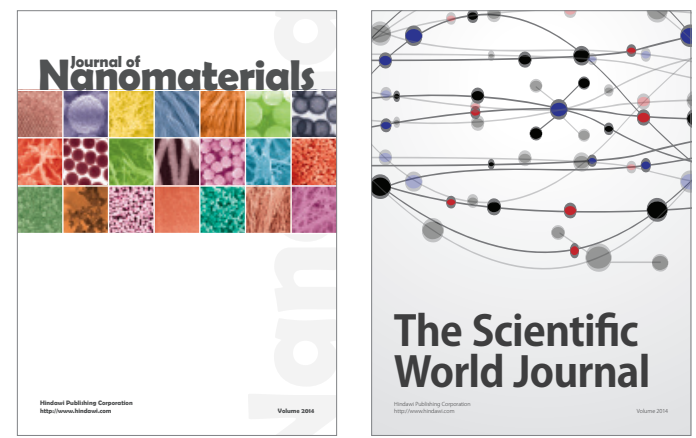

The Scientific World Journal
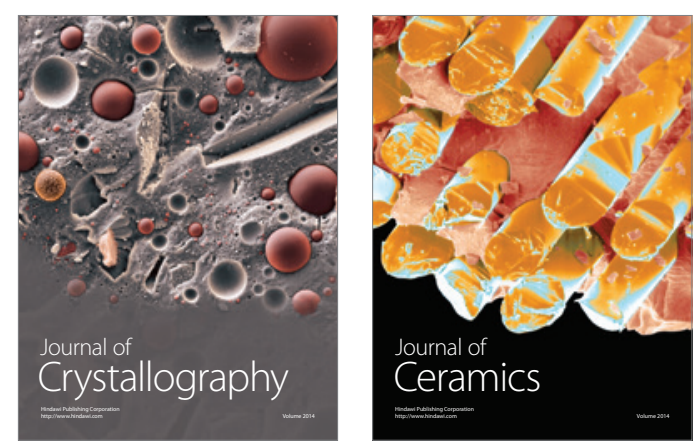
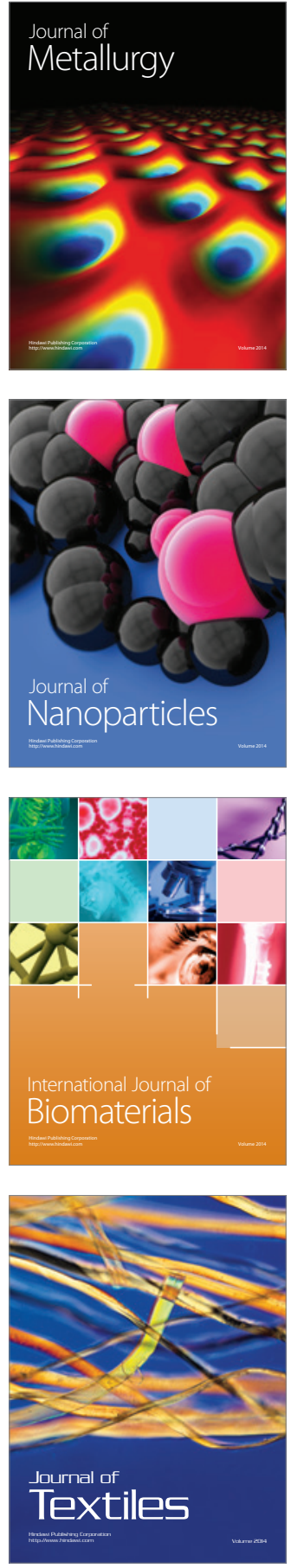